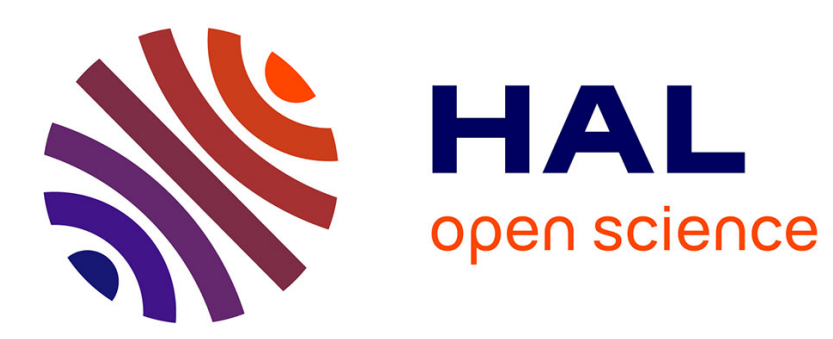

\title{
Steinberg's torsion theorem in the context of groups of finite Morley rank
}

\author{
Adrien Deloro
}

\section{To cite this version:}

Adrien Deloro. Steinberg's torsion theorem in the context of groups of finite Morley rank. Journal of Group Theory, 2009, 12 (5), pp.709-710. 10.1515/JGT.2009.005 . hal-01303301

\section{HAL Id: hal-01303301 \\ https://hal.science/hal-01303301}

Submitted on 17 Apr 2016

HAL is a multi-disciplinary open access archive for the deposit and dissemination of scientific research documents, whether they are published or not. The documents may come from teaching and research institutions in France or abroad, or from public or private research centers.
L'archive ouverte pluridisciplinaire HAL, est destinée au dépôt et à la diffusion de documents scientifiques de niveau recherche, publiés ou non, émanant des établissements d'enseignement et de recherche français ou étrangers, des laboratoires publics ou privés. 


\title{
Steinberg's torsion theorem in the context of groups of finite Morley rank
}

\author{
Adrien Deloro \\ (Communicated by A. V. Borovik)
}

\begin{abstract}
Groups of finite Morley rank generalize algebraic groups; the simple ones have even been conjectured to be algebraic. Parallel to an ambitious classification program towards this conjecture, one can try to show direct equivalents of known results on algebraic groups in the context of groups of finite Morley rank. This is done here with Steinberg's theorem on centralizers of semi-simple elements.
\end{abstract}

Theorem (cf. [5, Corollary 2.16(b)]). Let $G$ be a connected group of finite Morley rank of $p^{\perp}$-type and $\zeta \in G$ a p-element such that $\zeta^{p^{n}} \in Z(G)$. Then $C(\zeta) / C^{\circ}(\zeta)$ has exponent dividing $p^{n}$.

The argument is essentially different from Steinberg's methods. We shall need but little material; algebraically speaking the main tool is the connectedness of centralizers of tori.

The instruments. A general reference on groups of finite Morley rank is [2], where the reader will find everything needed here. $p^{\perp}$-type means that $G$ has trivial $p$-unipotent subgroups, which by definition are definable, connected, nilpotent $p$-groups of bounded exponent. This mimics a characteristic-not- $p$ assumption, and [3, Theorem 4] then yields conjugacy of Sylow $p$-subgroups, which are toral-by-finite, that is, finite extensions of $p$-tori. (Conjugacy is not known to hold in general.)

A $p$-torus is a finite direct sum of Prüfer $p$-groups $\mathbb{Z}_{p^{\infty}}$. Still about torality, [3, Corollary 3.1] shows that a $p$-element of a connected group of $p^{\perp}$-type is in any maximal $p$-torus of its centralizer. Decent tori are definable hulls of abelian, divisible, torsion groups. As their centralizers in a connected group are connected ([1, Theorem 1]; see also [4, Corollary 3.9]), so are those of $p$-tori.

On a more methodological side, Frattini arguments are corollaries of conjugacy theorems familiar to finite group theorists: if $H \unlhd G$ is a normal subgroup and $K \leqslant H$ a subgroup for which $H$ controls $G$-conjugacy, then $G=H \cdot N_{G}(K)$. This will be used repeatedly. 
So will actions. If $\sigma$ normalizes a $p$-torus $T$, then considering the map $t \mapsto[t, \sigma]$ in view of the underlying notion of dimension (the Prüfer rank, here), one easily finds that $T=C_{T}(\sigma)+[T, \sigma]$ with finite intersection. If $\sigma$ has order prime to $p$, the sum is clearly direct.

The argument. Let $k=p^{n}$. By torality there is a maximal $p$-torus $S$ with $\zeta \in S$. A Frattini argument implies that $C(\zeta)=C^{\circ}(\zeta) \cdot N_{C(\zeta)}(S)$, so lifting torsion [1, Fact 2.5] it suffices to show that if $\alpha$ is a $q$-element of $N_{C(\zeta)}(S)$ for any prime number $q$, then $\alpha^{k} \in C^{\circ}(\zeta)$.

If $q \neq p$, we have observed that $S=C_{S}(\alpha) \oplus[S, \alpha]$; in particular the subgroup $C_{S}(\alpha) \simeq S /[S, \alpha]$ is connected. Letting $T=C_{S}^{\circ}(\alpha)$, one therefore has $\zeta \in T$ and $\alpha \in C(T)=C^{\circ}(T) \leqslant C^{\circ}(\zeta)$. So we may assume that $q=p$.

By a Frattini argument, $\zeta$ normalizes a maximal $p$-torus $\Sigma$ of $C^{\circ}(\alpha)$; by torality $\alpha \in \Sigma$. One has $\Sigma=C_{\Sigma}(\zeta)+[\Sigma, \zeta]$; as the intersection is finite, one even has $\Sigma=C_{\Sigma}^{\circ}(\zeta)+[\Sigma, \zeta]$. Let $T=C_{\Sigma}^{\circ}(\zeta)$, and write $\alpha=\alpha_{1}+\alpha_{2}$ with $\alpha_{1} \in T, \alpha_{2} \in[\Sigma, \zeta]$. Let $\operatorname{Tr}$ denote the norm under the action of $\langle\zeta\rangle: \operatorname{Tr}(x)=x+x^{\zeta}+\cdots+x^{\zeta^{k-1}}$. Then a quick computation yields

$$
\alpha^{k}=\operatorname{Tr}(\alpha)=\operatorname{Tr}\left(\alpha_{1}\right)+\operatorname{Tr}\left(\alpha_{2}\right)=\alpha_{1}^{k}+0=\alpha_{1}^{k},
$$

so $\zeta \in C(T)=C^{\circ}(T) \leqslant C^{\circ}\left(\alpha_{1}^{k}\right)=C^{\circ}\left(\alpha^{k}\right)$.

But for $p$-elements $\mu$ and $v$ in a connected group of $p^{\perp}$-type, we have $\mu \in C^{\circ}(v)$ if and only if $v \in C^{\circ}(\mu)$ : each is equivalent to having a $p$-torus containing both $\mu$ and $v$. It follows $\alpha^{k} \in C^{\circ}(\zeta)$, and we are done.

Acknowledgments. Spasibo to Alexandre Borovik, and çok teşekkürler to Ali Nesin. The argument was found during the opening week of his Matematik Köyü at Şirince, a wonderful place for mathematics.

\section{References}

[1] T. Altınel and J. Burdges. On analogies between algebraic groups and groups of finite Morley rank. J. London Math. Soc. (2), 78 (2008), 213-232.

[2] T. Altınel, A. Borovik and G. Cherlin. Simple groups of finite Morley rank (American Mathematical Society, 2008).

[3] J. Burdges and G. Cherlin. Semisimple torsion in groups of finite Morley rank. Submitted (2008).

[4] O. Frécon. Pseudo-tori and subtame groups of finite Morley rank. J. Group Theory 12 (2009), 305-315.

[5] R. Steinberg. Torsion in reductive groups. Adv. Math. 15 (1975), 63-92.

Received 26 July, 2008; revised 11 November, 2008

Adrien Deloro, Rutgers University, Department of Mathematics, Hill Center, Busch Campus, 110 Frelinghuysen Road, Piscataway, NJ 08854, U.S.A.

E-mail: adeloro@math.rutgers.edu 